DOI: https://doi.org/10.34069/RA/2021.8.03

Volumen 4, Número 8/julio-diciembre 2021

Fiol Cuenca, A., Verdecia Cruz, A., Aguilera Saborit, G., \& Olivero Herrera, M. (2021). Alternativa para la enseñanza del idioma inglés con fines profesionales en la carrera de Ingeniería en Minas. Revista Científica Del Amazonas, 4(8), $29-37$. https://doi.org/10.34069/RA/2021.8.03

\title{
Alternativa para la enseñanza del idioma inglés con fines profesionales en la carrera de Ingeniería en Minas
}

\section{Alternative in the teaching of English language for professional purposes in Mining Engineering major}

\author{
Recibido: 18 de mayo de $2021 \quad$ Aceptado: 18 de julio de 2021
}

\author{
Autores: \\ Adis Fiol Cuenca ${ }^{14}$ \\ Adelfa Verdecia Cruz ${ }^{15}$ \\ Georgina Aguilera Saborit ${ }^{16}$ \\ Mirtha Olivero Herrera ${ }^{17}$
}

\section{Resumen}

En este trabajo se presenta una alternativa para la enseñanza del idioma inglés con fines profesionales en el contexto de la carrera de Ingeniería en Minas, en correspondencia con las nuevas exigencias derivadas del perfeccionamiento del proceso de enseñanza-aprendizaje del inglés en las universidades cubanas. Los materiales didácticos que se presentan constituyen la vía posible para solucionar los problemas que aún subsisten con respecto al aprendizaje del idioma inglés con fines profesionales, la motivación por el mismo y al desempeño comunicativo de los estudiantes, lo que sin dudas favorece la formación de un profesional más competente.

Palabras claves: asignatura electiva, material didáctico, programa analítico.

\section{Abstract}

This paper carries out an alternative to teach English language for professional purposes in the context of Mining Engineering major, in correspondence with the new demands derived from the improvement of English teaching-learning process in Cuban universities. The didactic materials presented are the possible way to solve the remaining problems regarding the learning of English language for professional purposes, the students 'motivation and their communicative performance which undoubtedly favors the formation of a more competent professional.

Key words: didactic material, elective subject, syllabus.

\footnotetext{
${ }^{14}$ Licenciada en Lenguas Extranjeras. Profesora Auxiliar. Decana de la Facultad de Ciencias Económicas. Master en Educación Superior. Profesora del Departamento de Idiomas, Universidad de Moa, Holguín, Cuba, https://orcid.org/0000-0002-0617-7515

15 Licenciada en Lenguas Extranjeras. Profesora Asistente. Master en Educación Superior. Profesora del Departamento de Idiomas, Universidad de Moa, Holguín, Cuba, https://orcid.org/0000-0003-4227-925X

${ }^{16}$ Licenciada en Lenguas Extranjeras. Vicedecana Docente de la Facultad de Ciencias Económicas. Profesora Auxiliar. Master en Educación Superior. Profesora del Departamento de Idiomas, Universidad de Moa, Holguín, Cuba, https://orcid.org/0000-0002-98555236

${ }^{17}$ Licenciada en Lenguas Extranjeras. Profesora Auxiliar. Master en Educación Superior. Jefa del Departamento de Idiomas. Universidad de Moa, Holguín, Cuba, https://orcid.org/0000-0002-8221-4410
} 


\section{AMAZONANAS}

\section{Introducción}

Las particularidades de las relaciones con el mundo del trabajo en la actualidad, establece determinadas exigencias al profesional contemporáneo, para quien resulta de vital importancia la actualización de los saberes en relación con la labor que desempeña. De ahí que la habilidad de leer y comunicarse en una lengua extranjera, es de gran utilidad, pues le permitirá apropiarse de la información que necesita, dentro de la amplia variedad de publicaciones que se editan a nivel mundial.

Lo anterior adquiere especial significación para el egresado universitario cubano, a partir del proceso de reestructuración del modelo económico del país y el establecimiento de nuevas relaciones académicas, científicas y laborales a nivel internacional, para lo cual resulta necesario el dominio de lenguas extranjeras, especialmente el inglés en su carácter de lengua de mayor uso y difusión para fines comunicativo, académico y profesional.

Ello se complementa con la proyección de desarrollo e inserción de la sociedad cubana en las relaciones económicas y comerciales a nivel global, lo cual demanda la formación de una comunidad universitaria bilingüe, apta para incursionar con éxito en este contexto.

En tal sentido, el Ministerio de Educación Superior (MES), propone una nueva estrategia para la enseñanza del idioma inglés en las universidades cubanas, para lo cual adopta el Marco Común Europeo de Referencia para las Lenguas (MCERL) como la base común que orienta la política nacional respecto al diseño de estándares, cursos de idioma inglés como lengua extranjera (cursos presenciales y/o virtuales), metodologías de enseñanza y materiales didácticos, e instrumentos de evaluación.

El MCERL establece una escala de seis niveles comunes de referencia para la organización del aprendizaje de lenguas:

$$
\begin{aligned}
& \text { Básico (A1 y A2) } \\
& \text { Intermedio (B1 y B2) } \\
& \text { Avanzado (C1 y C2) }
\end{aligned}
$$

Para llevar a cabo el Proceso de Enseñanza-Aprendizaje (PEA) del inglés se utiliza como bibliografía básica, entre otros, el libro seriado Face2Face, cuyo diseño posibilita el fortalecimiento de las cuatro habilidades lingüísticas, a saber: expresión oral, comprensión auditiva, lectura y escritura en diversos contextos comunicativos.

Sin embargo, aún queda por solucionar el problema de cómo lograr en los estudiantes un nivel en el uso y manejo de la lengua en el contexto profesional minero que les propicie un mejor desempeño en su campo de acción, de modo que el egresado pueda comunicarse e interactuar de forma oral y escrita con otros colegas en inglés y mantenerse actualizados desde lo académico y lo profesional sobre los avances en el campo de la minería, a través del uso de las tecnologías, la literatura propia de su especialidad y de otras formas de comunicación.

Para ello, es necesario garantizar las condiciones desde el punto de vista docente y metodológico de manera que el estudiante pueda contar con los recursos necesarios dirigidos a la consecución del fin antes expuesto.

En este contexto, se entiende como condiciones docentes la concepción de un plan para el aprendizaje a partir del diseño del programa de una asignatura basado en la teoría curricular, y motivado por los objetivos propuestos en función del proceso de formación del estudiante en la carrera. 
De ahí que como posible solución a esta problemática, se presenta una propuesta metodológica, que consiste en desarrollar el proceso de enseñanza-aprendizaje del Inglés con Fines Profesionales (IFP) como asignatura electiva en el diseño del Plan de estudio de la carrera de Minas.

\section{Marco Teórico}

\section{Fundamentación teórica de la propuesta.}

Asumir el aprendizaje del inglés con Fines Profesionales a partir de su concepción como asignatura electiva, presupone tener en cuenta los fundamentos teórico metodológicos que sustentan la propuesta como una estructura funcional que se inserta armónicamente en el currículo de la carrera, donde categorías como programa analítico, materiales didácticos y relaciones interdisciplinarias adquieren especial significación.

La programación de la formación académica de cualquier nivel de enseñanza, área de conocimiento o asignatura se materializa en el currículo y sus elementos componentes: el perfil profesional, el plan de estudios y los programas docentes.

El currículo se diseña a partir de la concepción de la carrera universitaria como un sistema que tiene como propósito común la formación del profesional donde se identifican diferentes subsistemas organizados en un orden jerárquico horizontal y vertical que parte de la carrera, luego el año académico, la disciplina, asignatura, tema, clase y por último la tarea docente. Así, por ejemplo, la signatura se subordina al año académico desde el punto de vista horizontal y a la disciplina a la que pertenece desde el vertical.

En cuanto a los contenidos que se estudian en la carrera, según se establece en el artículo 79 de la Resolución 2/2018 del Ministerio de Educación Superior Cubana (MES, 2018, p. 676), se organizan en tres tipos de currículo: base, propio y optativo/electivo.

Los contenidos del currículo base son de obligatorio cumplimiento para todas las instituciones de educación superior del país que desarrollan la carrera.

«A partir del currículo base, de los objetivos generales formulados para la carrera y de los programas de las disciplinas, el colectivo de la carrera de cada institución de educación superior decidirá cómo completar su plan de estudio particular (currículo propio y optativo/electivo), en correspondencia con sus características y la de cada territorio» (MES, 2018, p. 676)

En relación con las asignaturas electivas, se precisa en el artículo 81: «Las asignaturas electivas son aquellas que el estudiante elige libremente de acuerdo con sus gustos e intereses personales, a partir de un grupo de ofertas que se brindan y que pueden inclusive, pertenecer a otras carreras» (MES, 2018, p. 676).

Estas asignaturas podrán variar según las necesidades educativas, territoriales o las impuestas por el desarrollo científico técnico.

De acuerdo con Castañeda Hevia (2006, p.244), «el programa de una disciplina o asignatura constituye la descripción sistemática y jerárquica de los objetivos instructivoprofesionales, educativos y de formación de valores que se deben alcanzar en ella a partir y dentro de las definiciones dadas en el Modelo del profesional y el Plan de estudio, de los contenidos esenciales que este debe enseñar a los estudiantes, de los métodos y medios de enseñanza fundamentales, así como de los aspectos de organización en que se debe estructurar dicha 


\section{AMAZONANAS}

disciplina o asignatura para dar respuesta a los objetivos asignados a esta en el Modelo del profesional y en el plan de estudio».

Según el artículo 84 (MES, 2018, p. 676) de la resolución antes mencionada, los programas analíticos de las asignaturas deben contener, al menos, la información siguiente:

a) Datos generales (nombre de la asignatura, de la disciplina y de la carrera; su ubicación en el plan de estudio; el fondo de tiempo total y por formas organizativas; así como, la tipología de clases).

b) Objetivos generales de la asignatura.

c) La relación de temas, definiéndose para cada uno: los objetivos, el contenido, la cantidad de horas y su distribución por formas organizativas y tipos de clase, y la evaluación.

d) Indicaciones metodológicas y de organización.

e) El sistema de evaluación del aprendizaje.

f) Texto básico y otras fuentes bibliográficas.

La literatura docente resulta de vital importancia para asegurar el funcionamiento de la carrera como un sistema. «El libro, con independencia del programa de la asignatura, determina en la práctica el nivel de profundidad del contenido y con ello se establece una lógica que los estudiantes deben seguir en su trabajo independiente». (Horroutinier, 2008, p.45)

El libro de texto se revela como una variante de material didáctico esencial en la impartición de una asignatura. Jiménez Rosique (2009, p.25) plantea que «los materiales didácticos son instrumentos indispensables en la formación académica, proporcionan información y guían el aprendizaje, aportan una base concreta para el pensamiento conceptual y contribuyen al aumento de los significados, desarrollan la continuidad de pensamiento, hacen que el aprendizaje sea más duradero y brindan una experiencia real que estimula, la actividad de los estudiantes, proporcionan, además, experiencias que se obtienen fácilmente mediante diversos materiales y medios y ello ofrece un alto grado de interés para los alumnos; evalúan conocimientos y habilidades, así como proveen entornos para la expresión y la creación».

La creación de los materiales, dentro de los que se incluyen los libros de texto, cuadernos de ejercicios o cualquier otro que provea conocimiento de la lengua objeto de aprendizaje, muestra el esfuerzo y creatividad del diseñador o profesor. En ese sentido, el uso de materiales auténticos ofrece una visión del mundo del trabajo en el que los estudiantes se verán involucrados una vez graduados. Al mismo tiempo, la experiencia docente en la impartición de estos cursos ha demostrado su utilidad para elevar la motivación hacia el aprendizaje.

La selección del contenido en los cursos de inglés con fines profesionales depende de diversos factores. En primer lugar, resulta necesario tener presente las características propias de este tipo de curso definidas por Dudley-Evans \& St. John, (1998, p.4).

Estos autores consideran dos grupos de características: absolutas, entre las que se encuentran: estar dirigido a satisfacer determinadas necesidades específicas de los aprendices; utilizar la metodología y actividades de la disciplina a la que sirve y por último, centrarse en el lenguaje apropiado para estas actividades, en términos de la gramática, el léxico, el registro, las habilidades, el discurso y géneros de comunicación. Por otro lado, también se encuentran las características variables, a saber: relacionarse o diseñarse para disciplinas específicas; utilizar en determinadas situaciones de aprendizaje una metodología diferente a la del inglés general; Se diseña para diversos niveles de enseñanza o en contextos laborales y asume algunos conocimientos básicos del sistema de la lengua. 
El análisis de las necesidades de los estudiantes desde la perspectiva de la comunicación constituye el punto de partida para el diseño del programa del curso y la determinación de los contenidos junto al resto de las categorías didácticas: objetivos, métodos, medios y formas de evaluación, y en el inglés con fines profesionales estas necesidades se derivan de los objetivos que se plantea la carrera en el currículum con respecto a los problemas profesionales a resolver con el concurso de las habilidades comunicativas desarrolladas.

El planteamiento anterior conduce, al establecimiento de relaciones interdisciplinarias con otras asignaturas del currículo, al incursionar, desde los contenidos, en temáticas propias del ejercicio profesional. Ello significa que en el diseño del curso se deben tener en cuenta los contenidos de asignaturas que constituyen núcleos de conocimiento de la profesión en la carrera, lo cual determina su relevancia en la especialización de los estudiantes para un desempeño laboral más efectivo.

El elemento esencial de la interdisciplinariedad que se ha tenido en cuenta en la selección de los temas está dado por los nexos o vínculos de interrelación y cooperación entre las asignaturas debido a objetivos comunes que subyacen en los problemas profesionales del perfil minero. Por ello, en la formación del profesional de la minería, este vínculo se convierte en un elemento que no puede darse al margen de la profesionalidad, pues revela el principio interdisciplinario profesional que dirige el proceso docente educativo hacia la preparación de un profesional capaz de solucionar holísticamente los problemas que enfrentará en su futuro desempeño profesional.

\section{Propuesta de la asignatura electiva inglés con Fines Profesionales en la carrera de Ingeniería en Minas.}

\section{Programa analítico}

El programa analítico es el documento que describe el modelo del proceso docente educativo de la asignatura y se materializa con la participación consciente del profesor y del estudiante.

La asignatura electiva inglés con Fines Profesionales se ubica en el 3er año de la carrera con una cantidad de 40 horas lectivas en la modalidad presencial.

Los objetivos generales educativos e instructivos expresan la transformación planificada que se desea lograr en los estudiantes, orientando así el trabajo de profesores y estudiantes en el proceso.

Los objetivos instructivos se expresan en términos de aprendizaje y los objetivos educativos están formulados en términos de la profesión, de manera que el profesor pueda conducir las acciones necesarias para el cumplimiento de estos en el proceso de enseñanzaaprendizaje.

El cumplimiento de los objetivos, ¿se materializa a partir de la interrogante qué enseñar?

En consecuencia, el contenido de la asignatura es aquella parte que debe ser asimilado por el estudiante para alcanzar los objetivos previstos y se agrupa en un sistema de conocimientos y de habilidades en función de la formación profesional del estudiante. criterios:

Para la selección de los contenidos de la asignatura se tuvieron en cuenta los siguientes

- Las necesidades de aprendizaje de los estudiantes en el contexto académico-profesional. 


\section{AMAZÉNAS}

- La selección de los contenidos en vínculo directo con la actividad profesional de los estudiantes.

- El establecimiento de relaciones interdisciplinarias con asignaturas pertenecientes a los currículos base y propio de la carrera.

- La consideración de la interacción y la colaboración como formas de realización de la actividad de aprendizaje,

- La planeación de una evaluación estimuladora y formativa, en tanto que posibilite la comprensión del estudiante de sus propias dificultades y asuma conscientemente cómo superarlas.

Los contenidos a impartir en la asignatura se vinculan estrechamente con las asignaturas pertenecientes a los currículos base y propio de la carrera mediante el establecimiento de nexos de colaboración interdisciplinaria entre los departamentos de Idiomas y Minas.

Los temas seleccionados se centran en contenidos específicos del ejercicio profesional relacionados con:

- History of mining

- Surveying

- Basic concepts and mining terminology

- Surface mining

- Underground mining

- Mining and environment

Las indicaciones metodológicas y de organización tienen especial significación pues aquí se expresa la lógica interna del proceso docente educativo para la adquisición de conocimientos y el establecimiento del vínculo entre las categorías objetivo, contenido, método, medios, formas organizativas y evaluación del aprendizaje; además orienta cómo satisfacer las necesidades educativas y de formación de valores de los estudiantes a través de la actividad docente.

En la concepción de las indicaciones metodológicas también se tiene en cuenta el enfoque con que se debe dirigir el trabajo independiente del estudiante para lograr un aprendizaje consciente y las formas para comprobar sistemáticamente el progreso en la asimilación de los contenidos.

La evaluación del aprendizaje es un componente del PEA de gran importancia, se utiliza la evaluación parcial dirigida a comprobar el cumplimiento de los objetivos particulares de los temas. La evaluación final se realiza a través de un trabajo de curso integrador con la finalidad de comprobar el cumplimiento de los objetivos generales de la asignatura y su vínculo con otras asignaturas de la carrera.

El programa no puede verse simplemente como un documento donde se recogen los contenidos a asimilar por los estudiantes, sino que se convierte en un documento metodológico de gran valor para el profesor, puesto que en él se plasman con exactitud el porqué de la existencia de la asignatura; para qué se enseña; qué se enseña; cómo debe desarrollar el proceso para lograr el aprendizaje consciente del estudiante; cómo comprobar el cumplimiento de los objetivos; cómo lograr la interrelación de los contenidos y cómo mediante la instrucción se puede contribuir al desarrollo de la personalidad del estudiante. 


\section{AMAZÓNAS}

\section{Materiales didácticos}

Material didáctico es el medio o recurso cuyo empleo facilite la adquisición de conceptos, habilidades, actitudes y destrezas en el proceso de enseñanza- aprendizaje. En el caso específico del inglés con Fines Profesionales en la carrera de Ingeniería de Minas se puede definir material didáctico como cualquier fuente que se pueda utilizar para ayudar a los estudiantes en el proceso de aprendizaje del inglés en el contexto comunicativo minero.

Dentro de los materiales didácticos elaborados se encuentran:

Folleto de selección de lecturas constituye el texto básico de la asignatura. Entre sus características más relevantes se destaca:

En su diseño se han tenido en cuenta artículos auténticos escritos en idioma inglés por investigadores reconocidos, tanto en el ámbito nacional como internacional.

- Se presentan contenidos actualizados relacionados con el perfil de los estudiantes de la carrera de Ingeniería en Minas. De esta forma no solo se garantiza el aprendizaje de los fundamentos de lengua, sino también la vinculación entre el idioma inglés y el resto de las asignaturas de la carrera.

- Los contenidos teóricos que se presentan posibilitan la comprensión de la información en dos direcciones: como parte del desarrollo del proceso de enseñanza-aprendizaje en el aula y como vía para la autopreparación de los estudiantes, para reforzar los contenidos estudiados mediante la solución de tareas en las que la comprensión auditiva y lectora y expresión oral resultan fundamentales.

- En la concepción de los materiales también se considera la doble función de instrumento de trabajo y cultura que cumple el aprendizaje del inglés y su vinculación con otras disciplinas de la carrera.

- Se prioriza una variedad de ejercicios con el fin de promover el interés por el aprendizaje de la lengua y la incorporación del léxico específico a través de tareas comunicativas.

- Se tiene en cuenta el desarrollo armónico de las habilidades lingüísticas para fortalecer las posibilidades de comunicación de los estudiantes desde el punto de vista académico y profesional.

Como complemento del folleto se confeccionó un set de láminas que tiene como propósito sistematizar los contenidos de la selección de lecturas y las habilidades de escritura y expresión oral.

A continuación se muestran ejemplos de las láminas.

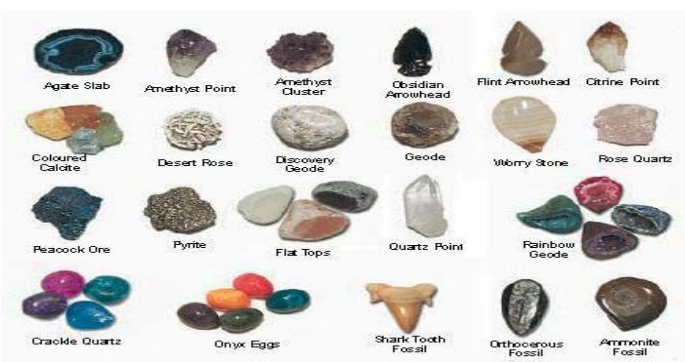

Minerales

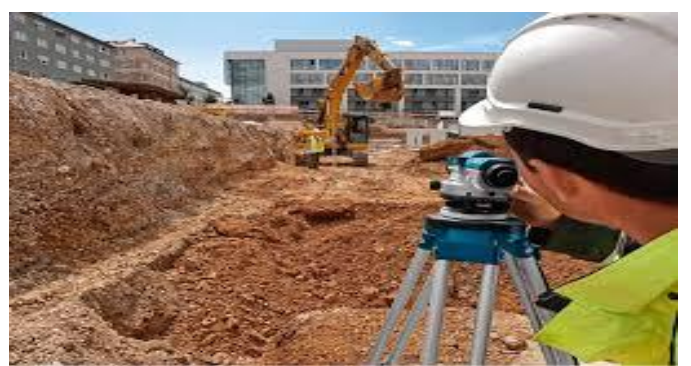

Topógrafo 


\section{AmAZGNÁNAS}

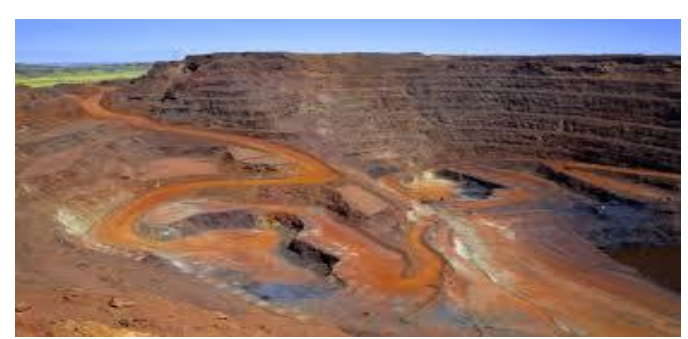

Mina a cielo abierto

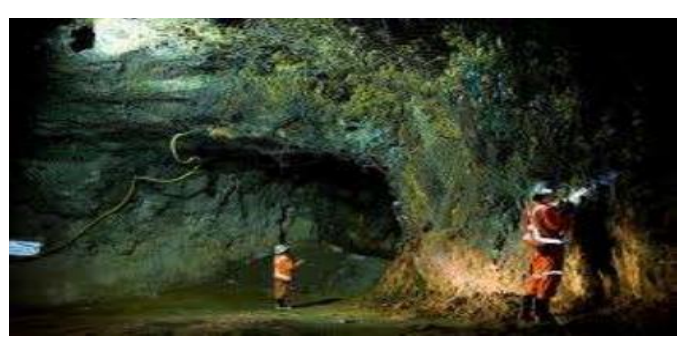

Mina subterránea

Para una mejor comprensión de la literatura publicada en idioma inglés en el campo de la minería se elaboró un glosario de términos inglés español que facilita un mejor entendimiento del discurso científico.

Glosario de términos mineros inglés - español. Favorece el desarrollo de habilidades en la obtención de información científica en inglés, contribuye a elevar el grado de independencia en la adquisición de información y suple el déficit de diccionarios y glosarios bilingüe en esta temática.

\section{Resultados}

El diseño y la puesta en práctica del Idioma Inglés con Fines Profesionales como asignatura electiva en la carrera de Ingeniería en Minas permiten:

- Incremento de la motivación de estudiantes y profesores por el estudio del idioma con fines profesionales.

- Mayor vinculación interdisciplinaria con las temáticas científico-técnicas de la carrera.

- El trabajo con textos de contenido técnico especializado sobre diferentes temas curriculares dirigidos a la obtención de información y el léxico necesario para la producción oral, así como la vinculación de los contenidos con la realidad profesional contribuyen a elevar la motivación de los estudiantes, por constituir un elemento esencial para garantizar la competitividad en el mercado laboral.

- Las actividades diseñadas para el uso de los materiales didácticos son expresión del aporte de la enseñanza del inglés como lengua extranjera a la formación del modo de actuación del futuro profesional de la minería.

\section{Conclusiones}

El diseño de la asignatura Inglés con Fines Profesionales como asignatura electiva en el proceso de enseñanza y aprendizaje en la carrera de Ingeniería en Minas posibilita desarrollar en los estudiantes un nivel de comunicación oral y escrita y de comprensión auditiva y lectora que le permita al futuro egresado poder comunicarse con profesionales de su rama e intercambiar, a través del uso de las tecnologías, de la literatura propia de su especialidad y de otras formas de comunicación en correspondencia con los avances en el campo de la minería.

Los temas seleccionados, proveen al estudiante de una fuente de información funcional, desde el punto de vista de la adquisición de conocimientos de interés comunicativo profesional, para un eficiente desarrollo del proceso de enseñanza aprendizaje del idioma inglés y la adquisición de las técnicas y hábitos de acceso a la información científico-técnica como vía de acceder a los conocimientos de la especialidad.

La conceptualización de términos específicos relacionados con la minería, facilita la consulta de bibliografía en inglés, posibilitando a su vez la profundización en temas de interés 
profesional y un amplio acceso al conocimiento de los últimos adelantos de la ciencia y la tecnología.

\section{Referentes Bibliográficas}

Castañeda, A. (2006). La asignatura como parte de un Sistema de mayor jerarquía. Aspectos conceptuales básicos vinculados al currículo y al diseño curricular, en Preparación Pedagógica integral para profesores integrales. La Habana: Ed. Felix Varela, pp. 192

Dudley, E. y St. John. (1998). Developments in English for specific purposes: A multidisciplinary approach. Cambridge: Cambridge University Press, p. 4.

Fiol, A y Aguilera G. (2019). La enseñanza del idioma inglés con fines profesionales en la carrera de ingeniería en minas. Cuadernos de Educación y Desarrollo, issue 112. https://www.eumed.net/rev/atlante. (7/1/2021)

Horroutinier, P. (2008). La Universidad cubana: el modelo de formación. La Habana: Ed. Felix Varela, pp. 45.

Jiménez, R. (2009). La Importancia del material didáctico en el proceso de enseñanzaaprendizaje. http://www.monografía.com. (12/12/2020).

Morgan, M. (2019). Concepción didáctica de la integración de las habilidades en la enseñanza del idioma inglés con fines específicos. Formación y Calidad Educativa (REFCaLE), Vol.7, No.2, www.refcale.uleam.edu.ec. (19/11/2020)

Resolución 2. GOC-2018-460-025. Reglamento docente metodológico. Gaceta Oficial de la República de Cuba. Ministerio de Justicia. Edición ordinaria, No 25, pp. 647. Ministerio de Educación Superior, 2018. 